\title{
Wigner Distribution Analysis of a Schrödinger Cat Superposition of Displaced Equilibrium Coherent States
}

\author{
G.W. Ford $\dagger$ and R.F. O’Connellł* \\ School of Theoretical Physics, Dublin Institute for Advanced Studies \\ 10 Burlington Road, Dublin 4, Ireland
}

(November 10, 2017)

\begin{abstract}
Motivated by recent experiments, we consider a Schrödinger cat superposition of two widely separated coherent states in thermal equilibrium. The time development of our system is obtained using Wigner distribution functions. In contrast to our discussion for a two-Gaussian wave packet [Phys. Lett. A 286 (2001) 87], we find that, in the absence of dissipation, the interference term does not decay rapidly in time, but in common with the other two terms, it oscillates in time and persists for all times.
\end{abstract}

${ }^{*}$ Corresponding Author:

e-mail:rfoc@rouge.phys.lsu.edu (R. F. O'Connell) 
Decoherence phenomena are at the forefront of cutting-edge research in fundamental and applied quantum mechanics, as summarized in [1], where we stressed that, at variance with common lore, decoherence without dissipation could occur in the case of a prototypical Schrödinger cat state of two widely separated Gaussians. The question arises as to whether this is a general phenomena so here, motivated by the NIST experiments on trapped ions $[2,3]$, we examine decoherence without dissipation for the case of a widely separated pair of equilibrium coherent states.

Consider any quantum state consisting of two identical components separated by a distance $d$. Then, it is not difficult to show that the corresponding Wigner distribution function, $W^{(2)}(q, p)$ at time $t=0$, say, is given by

$$
W^{(2)}(q, p, 0)=N_{0}\left\{W\left(q+\frac{d}{2}, p, 0\right)+W\left(q-\frac{d}{2}, p, 0\right)+2 \cos \left(\frac{p d}{\hbar}\right) W(q, p, 0)\right\}
$$

where $W(q, p, 0)$ is the Wigner function for one of the pairs at $t=0$ and $N_{0}$ is a normalization factor. We wish to examine the case where $W(q, p, 0)$ is an oscillator state and where $W\left(q \pm \frac{d}{2}, p, 0\right)$ corresponds to the pair of coherent (displaced oscillator) states.

Decoherence is a measure of how the interference term in (1) decays in time relative to the other terms. Thus, we need to calculate $W^{(2)}(q, p, t)$. This calculation is facilitated by the fact that the equation of motion for the Wigner function of an oscillator is the same as the classical equation of motion [4]. Hence

$$
W(q, p, t)=W(q(-t), p(-t), 0)
$$

where

$$
q(t)=q \cos \omega t+\left(\frac{p}{m \omega}\right) \sin \omega t
$$

and

$$
p(t)=p \cos \omega t-m \omega q \sin \omega t
$$

where $\omega$ is the oscillator frequency. It follows that 


$$
\begin{aligned}
W^{(2)}(q, p, t)=A & \left\{W\left(q(-t)+\frac{d}{2}, p(-t), 0\right)+W\left(q(-t)-\frac{d}{2}, p(-t), 0\right)\right. \\
+ & \left.2 \cos \left(\frac{p(-t) d}{\hbar}\right) W(q(-t), p(-t), 0)\right\},
\end{aligned}
$$

and $A$ is now the appropriate normalization factor.

We now consider the case where the system is in thermal equilibrium. The equilibrium Wigner function for the oscillator is [4]

$$
W_{0}(q, p)=\frac{1}{2 \pi m \sqrt{\left\langle\dot{q}^{2}\right\rangle\left\langle q^{2}\right\rangle}} \exp \left\{-\frac{p^{2}}{2 m^{2}\left\langle\dot{q}^{2}\right\rangle}-\frac{q^{2}}{2\left\langle q^{2}\right\rangle}\right\},
$$

where the subscript "0" indicates equilibrium and

$$
\left\langle\dot{q}^{2}\right\rangle=\omega^{2}\left\langle q^{2}\right\rangle=\frac{\hbar \omega}{2 m} \operatorname{coth}\left(\frac{\hbar \omega}{2 k T}\right)=\frac{\hbar \omega}{2 m}(2 N+1) .
$$

It follows from (3), (4), and (6) that

$$
W_{0}(q(-t), p(-t), 0)=W_{0}(q, p, 0) .
$$

Hence

$$
\begin{aligned}
W_{0}^{(2)}(q, p, t)=A_{0} W_{0} & (q, p, 0)\left(\exp \left\{-\frac{1}{2\left\langle q^{2}\right\rangle}\left[\frac{d^{2}}{4}+d\left(q \cos \omega t-\frac{p}{m \omega} \sin \omega t\right)\right]\right\}\right. \\
+ & \exp \left\{-\frac{1}{2\left\langle q^{2}\right\rangle}\left[\frac{d^{2}}{4}-d\left(q \cos \omega t-\frac{p}{m \omega} \sin \omega t\right)\right]\right\} \\
+ & \left.2 \cos \left\{\frac{(p \cos \omega t+m \omega q \sin \omega t) d}{\hbar}\right\}\right),
\end{aligned}
$$

where

$$
A_{0}=\left(2\left[1+\exp \left\{-\frac{m^{2}\left\langle\dot{q}^{2}\right\rangle d^{2}}{2 \hbar^{2}}\right\}\right]\right)^{-1} .
$$

As before, decoherence is given a quantitative measure by defining an attenuation coefficient $a_{w}(t)$, which is the ratio of the factor multiplying the cosine to twice the geometric mean of the first two terms (and here the subscript $w$ indicates decoherence in phase space to distinguish it from the more physically meaningful quantity $a(t)$ corresponding to decoherence in coordinate space). Hence 


$$
a_{w}(t)=\exp \left\{\frac{d^{2}}{8\left\langle q^{2}\right\rangle}\right\} .
$$

Next, we obtain the coordinate probability by integrating (9) over $p$. First, we note, using (6), that

$$
\begin{aligned}
P_{0}(q, 0) & =\int_{-\infty}^{\infty} W_{0}(q, p, 0) d p \\
& =\left[2 \pi\left\langle q^{2}\right\rangle\right]^{-1 / 2} \exp \left\{-\frac{q^{2}}{2\left\langle q^{2}\right\rangle}\right\} .
\end{aligned}
$$

It follows, from (9) and (12), that

$$
\begin{aligned}
P^{(2)}(q, t) & =A_{0} P_{0}(q, t)\left(\exp \left\{-\frac{1}{2\left\langle q^{2}\right\rangle}\left(\frac{d^{2}}{4} \cos ^{2} \omega t-q d \cos \omega t\right)\right\}\right. \\
& +\exp \left\{-\frac{1}{2\left\langle q^{2}\right\rangle}\left(\frac{d^{2}}{4} \cos ^{2} \omega t+q d \cos \omega t\right)\right\} \\
& \left.+2 \exp \left\{-\frac{m^{2}\left\langle\dot{q}^{2}\right\rangle d^{2}}{2 \hbar^{2}} \cos ^{2} \omega t\right\} \cos \left(\frac{d m \omega q \sin \omega t}{\hbar}\right)\right) .
\end{aligned}
$$

Hence

$$
\begin{aligned}
a(t) & =\exp \left\{\frac{d^{2}}{8\left\langle q^{2}\right\rangle} \cos ^{2} \omega t-\frac{d^{2} m^{2}\left\langle\dot{q}^{2}\right\rangle}{2 \hbar^{2}} \cos ^{2} \omega t\right\} \\
& =\exp \left\{-\frac{m \omega d^{2} \cos ^{2} \omega t}{2 \hbar \sinh \left(\frac{\hbar \omega}{k T}\right)}\right\} .
\end{aligned}
$$

It is clear that we do not have an interference term which decays rapidly in time but instead, in common with the other terms, it oscillates in time and persists for all time. This is in contrast to what we found for a two-Gaussian state of a free particle $[1,5]$. However, for small times after the times for which the attenuation factor has its maximum value of unity, and for negligibly small frequencies, we obtain a decoherence decay time which is consistent with our results for a free particle $[1,5]$. However, for small times after the times for which the attenuation factor has its maximum value of unity, and for negligibly small frequencies, we obtain a decoherence decay time which is consistent with our results for a free particle $[5]$. 


\section{REFERENCES}

$\dagger$ Permanent address: Department of Physics, University of Michigan, Ann Arbor, MI 48109-1120.

$\ddagger$ Permanent address: Department of Physics and Astronomy, Louisiana State University, Baton Rouge, LA 70803-4001.

[1] G. W. Ford and R. F. O'Connell, Am. J. Phys. 70 (2002) 319.

[2] C. J. Myatt, B. E. King, Q. A. Turchette, C. A. Sackett, D. Kielpinski, W. M. Itano, C. Monroe and D. J. Wineland, Nature 403 (2000) 269.

[3] O. A. Turchette, C. J. Myatt, B. E. King, C. A. Sackett, D. Kielpinski, W. M. Itano, C. Monroe and D. J. Wineland, Phys. Rev. A 62 (2000) 053807.

[4] M. Hillery, R. F. O'Connell, M. O. Scully and E. P. Wigner, Phys. Rep. 106 (1984) 121.

[5] G. W. Ford and R. F. O'Connell, Phys. Lett. A 286 (2001) 87. 